\title{
Effects of diesel exhaust particles on microRNA-21 in human bronchial epithelial cells and potential carcinogenic mechanisms
}

\author{
FANG ZHOU $^{1}$, SULI LI ${ }^{1}$, WENLIANG JIA ${ }^{1}$, GANG LV $^{2}$, CHONGLIN SONG $^{2}$, \\ CHUNSHENG KANG ${ }^{3}$ and QINGYU ZHANG ${ }^{1}$ \\ ${ }^{1}$ Department of Gastroenterology, Tianjin Medical University General Hospital, Tianjin 300052; \\ ${ }^{2}$ State Key Laboratory of Internal Combustion Engine Fuel Science of Tianjin University, Tianjin 300072; \\ ${ }^{3}$ Department of Neurosurgery, Tianjin Medical University General Hospital and Laboratory of Neuro-Oncology, \\ Tianjin Neurological Institute, Tianjin 300052, P.R. China \\ Received February 11, 2014; Accepted May 16, 2014
}

DOI: $10.3892 / \mathrm{mmr} .2015 .3655$

\begin{abstract}
Air pollution plays a role in cancer risk, particularly in lung cancer, which is the leading cause of cancer-related mortality worldwide. Diesel exhaust particles (DEPs), a component of diesel exhaust products, is a complex mixture of particle compounds that include a large number of known and suspected human carcinogens. Historically, lung cancer, which is associated with DEPs, has been the focus of attention as a health risk in human and animal studies. However, the mechanism by which DEPs cause lung cancer remains unclear. The present study reports that DEPs increased miR-21 expression and then activated the PTEN/PI3K/AKT pathway in human bronchial epithelial (HBE) cells, which may serve as an important carcinogenic mechanism. However, the data revealed that short-term exposure to a high DEP concentration did not cause evident cell carcinogenesis in HBE cells.
\end{abstract}

\section{Introduction}

Lung cancer is the first and second leading cause of cancer-related mortality in males and females, respectively, worldwide. In numerous regions of the world, the number of cases and deaths associated with lung cancer is on the rise (1), however, the etiological factors of the disease remain unknown. Early-stage lung cancer is curable and may be alleviated by radiation, chemotherapy, targeted therapies and surgical resection. However, lung cancer is diagnosed at advanced stages in the majority of patients (2); therefore, studies investigating the

Correspondence to: Dr Qingyu Zhang, Department of Gastroenterology, Tianjin Medical University General Hospital, 154 Anshan Road, Heping, Tianjin 300052, P.R. China

E-mail: zhangqy@tijmu.edu.cn

Key words: diesel exhaust particles, normal human bronchial epithelial cells, lung adenocarcinoma cells, microRNA-21, antisense oligonucleotide of microRNA-21, morphology etiology of lung cancer and its carcinogenic mechanisms are a primary focus in lung cancer studies.

Air pollution, primarily from fossil fuel combustion, is an important factor for the development of cancer. The combustion of diesel fuel is a likely source of air pollution that affects cancer risk on a large scale (3). Diesel exhaust particles (DEPs), a component of the diesel exhaust product, was classified as a 'probable' carcinogen by the International Agency for Research on Cancer (IARC) in 1989 (4). This classification was confirmed by a series of human and animal studies suggesting that DEPs have an important role in tumorigenesis in a variety of cancer types, including gastrointestinal, cervical, breast and particularly, lung cancer (4-8). However, the mechanism by which DEPs cause cancer remains unclear.

MicroRNAs (miRNAs) are single-stranded RNA 20-30 nucleotides in length that function as negative regulators to silence or suppress gene expression. Aberrant miRNA expression has been implicated in several cellular processes and the pathogenic pathways of a number of diseases (9). Initial studies in ovarian, breast, bladder and lung cancer patients demonstrated that the levels of specific miRNAs in cells are important with regard to the occurrence, development and treatment of the types of cancer (10-13).

Previous studies demonstrated that DEPs activate a variety of carcinogenic pathways (14). Additional studies have indicated that DEPs increase or decrease the expression of a variety of microRNAs (15). However, this finding is not a definitive result. In the present study, microRNAs were combined with carcinogenic pathways to examine the potential carcinogenic mechanism of DEPs in normal human bronchial epithelial (HBE) cells. It was confirmed that DEPs upregulate the expression of miR-21. DEPs activate phosphatase and tensin homolog (PTEN)/phosphatidylinositol 3'-kinase (PI3K)/AKT signaling through miR-21. The present study may provide a new mechanism for lung carcinogenesis and it is expected to serve as a reference for investigating lung cancer etiology.

\section{Materials and methods}

Cell culture. HBE cells were purchased from American Type Culture Collection (ATCC; Rockville, MD, USA). 
Lung adenocarcinoma cells (A549) were obtained from the Laboratory of Neuro-oncology, Tianjin Neurological Institute (Tianjin, China). The cells were cultured in RPMI-1640 supplemented with $10 \%$ fetal calf serum (FCS), $1 \%$ penicillin-streptomycin and $1 \%$ glutamine. All of the cells were cultured in six-well plates in a $37^{\circ} \mathrm{C}$ humidified incubator supplied with $5 \% \mathrm{CO}_{2}$.

DEP treatment. The DEPs were a gift from the State Key Laboratory of Internal Combustion Engine Fuel Science of Tianjin University (Tianjin, China). DEPs $(100 \mu \mathrm{g} / \mathrm{ml})$ were freshly prepared and suspended in $100 \mathrm{ml} \mathrm{RPMI}-1640$ with $10 \%$ FCS, $1 \%$ penicillin-streptomycin and $1 \%$ glutamine by sonication. The suspended particles were then stored at $4^{\circ} \mathrm{C}$ in the dark. The cells were cultured in six-well plates and then treated with $3 \mathrm{ml}$ RPMI-1640 in the experimental group.

Oligonucleotides and cell transfection with the 2'-O-methyl (2'-O-Me-) $m i R-21$. The inhibitors were chemically synthesized by Shanghai GenePharma (Shanghai, China). The 2'-O-Me oligonucleotides were composed entirely of 2'-O-methyl bases and contained the following sequences: 5'-GTCCAC TCT TGT CCT CAA TG-3'; scrambled sequences were 5'-AAG GCA AGC UGA CCC UGA AGU-3'. The oligonucleotides were purified using a high-pressure liquid chromatography system, dissolved in diethylpyrocarbonate (DEPC) water and then frozen at $-20^{\circ} \mathrm{C}$. Oligonucleotides $(50 \mathrm{~nm} / \mathrm{l})$ were transfected into HBE cells at $70 \%$ confluence using Oligofectamine according to the manufacturer's instructions (Invitrogen Life Technologies, Carlsbad, CA, USA).

RNA extraction and quantitative polymerase chain reaction ( $q P C R)$. Total RNA was extracted using the TRIzol reagent (Invitrogen Life Technologies) according to the standard procedures. A NanoDrop spectrophotometer (Gene Co., Ltd., Hong Kong, China) was used to detect the total RNA concentration. Total RNA ( $1 \mu \mathrm{g})$ was used to synthesize cDNA by reverse transcription with MMLV reverse transcriptase (Promega Corporation, Madison, WI, USA), according to the manufacturer's instructions. qPCR analysis was performed to determine the miR-21 expression in HBE cells, A549 and DEP-treated HBE cells $48 \mathrm{~h}$ following transfection with the miR-21 inhibitor or scrambled negative control. The expression of $\mathrm{u} 6$ was used as an internal control. A total of 40 cycles of qPCR were performed, consisting of $95^{\circ} \mathrm{C}$ for $3 \mathrm{~min}, 95^{\circ} \mathrm{C}$ for $15 \mathrm{sec}$ and $60^{\circ} \mathrm{C}$ for $30 \mathrm{sec}$. RT and PCR primers were purchased from GenePharma, $5 \mathrm{~S}$ was used for normalization. The relative quantification was conducted using amplification efficiencies derived from cDNA standard curves. The data are expressed as the fold change $\left(2^{-\Delta \Delta \mathrm{Ct}}\right)$ and initially analyzed using Opticon Monitor Analysis Software V2.02 software (MJ Research Inc., Waltham, MA, USA).

Protein extraction and western blotting. Following treatment with DEPs and DEPs in combination with the miR-21 antisense oligonucleotide (ASODN) for $48 \mathrm{~h}$, the adherent cells in the six-well plate were rinsed with phosphate-buffered saline (PBS), and the cell extracts were prepared by suspension in ice-cold lysis buffer. Floating cells from the same well were collected, lysed and mixed with SDS loading buffer $(100 \mathrm{mM}$
Tris-Cl, pH 6.8; 200 mM DTT; 4\% SDS; 0.2\% bro-P-40 lysis buffer [20 mM Tris, $\mathrm{pH} 8.0 ; 137 \mathrm{mM} \mathrm{NaCl} ; 1 \%$ Nonidet P-40; 10\% glycerol; $1 \mathrm{mM} \mathrm{CaCl2;} 1 \mathrm{mM} \mathrm{MgCl2} ; 1 \mathrm{mM}$ phenylmethylsulfonyl fluoride; $1 \mathrm{mM}$ sodium fluoride; $1 \mathrm{mM}$ sodium orthovanadate; and a protease inhibitor mixture]). Homogenates were clarified by centrifugation at $12,000 \mathrm{x} \mathrm{g}$ for $15 \mathrm{~min}$ at $4^{\circ} \mathrm{C}$, and protein concentrations were determined using a bicinchoninic acid protein assay kit (Pierce Biotechnology, Inc., Rockford, IL, USA). The next $40 \mathrm{mg}$ of lysates was subjected to SDS-PAGE on $8 \%$ SDS-acrylamide gels. The separate proteins were transferred onto PVDF membranes (Millipore, Bedford, MA, USA) and incubated with primary antibodies against AKT (1:1,000 dilution; rabbit polyclonal; Signalway Antibody, College Park, MD, USA), PI3K (1:1,000 dilution; mouse monoclonal; Cell Signaling Technology, Inc., Beverly, MA, USA), PTEN, caspase 3, PCNA, cyclin D1, Bcl-2 (1:100 dilution; mouse polyclonal; Zhongshan Goldenbridge Biotechnology Co., Ltd., Beijing, China) and GAPDH (1:100 dilution; rabbit polyclonal; Zhongshan Goldenbridge Biotechnology Co., Ltd.). The membranes were then incubated with an HRP-conjugated secondary antibody (Zymed Laboratories Inc., San Francisco, CA, USA). Specific proteins were detected using a Super Signal protein detection kit (Pierce Biotechnology, Inc.). The membrane was reprobed with an antibody against GAPDH (1:100 dilution; rabbit polyclonal; Zhongshan Goldenbridge Biotechnology Co., Ltd.) using the procedures described above.

Cell cycle analysis. For cell cycle analysis using FCM (flow cytometry), log phase transfected and control cells were harvested, washed with PBS, fixed with $90 \%$ ethanol overnight at $41^{\circ} \mathrm{C}$, then incubated with RNase at $37^{\circ} \mathrm{C}$ for $30 \mathrm{~min}$. The cell nuclei were stained with propidium iodide (PI) for an additional $30 \mathrm{~min}$. A total of 104 nuclei were examined using a FACS Calibur flow cytometer (Becton-Dickinson, Franklin Lakes, NJ, USA). The samples were analyzed using flow cytometry for the FL-2 area, DNA histograms were analyzed using Modifit software. The experiments were performed in triplicate. The results are presented as the percentage of cells in a given phase. The apoptosis assays were conducted using annexin staining and TUNEL methods.

Cells apoptosis analysis. To quantify DEP-induced apoptosis, annexin V/PI staining was performed, and apoptosis was evaluated by flow cytometry analysis. Following DEP treatment for $48 \mathrm{~h}$, the floating and attached cells were collected and subjected to annexin V/PI staining using an annexin V-FITC Apoptosis Detection kit (BioVision, Palo Alto, CA, USA), according to the manufacturer's instructions. The resulting fluorescence was measured by flow cytometry using a FACS flow cytometer (Becton-Dickinson).

Matrigel invasion assay. The invasive ability of treated and untreated HBE and A549 cells was assessed using a 24-well transwell chamber and a reconstituted extracellular matrix membrane (Matrigel, BD Biocoat; BD Bioscience Franklin Lakes, NJ, USA). The cell invasion chambers were prepared by placing $100 \mu \mathrm{l}$ of a 1:5 dilution of Matrigel onto the upper filter and then incubating the cells for $\sim 30 \mathrm{~min}$ at $37^{\circ} \mathrm{C}$ to allow for Matrigel polymerization. The untreated HBE and A549 cells, 
A

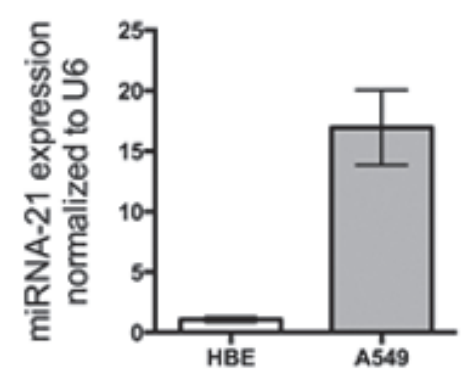

B

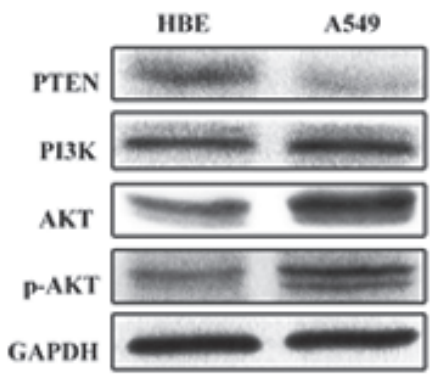

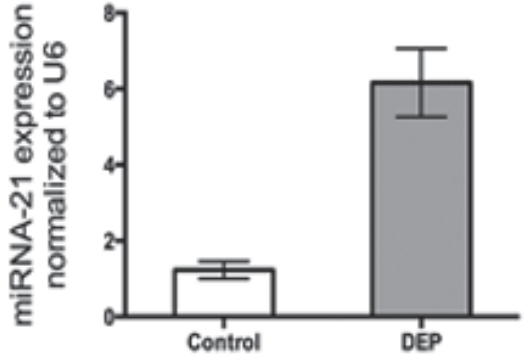

C

HBE

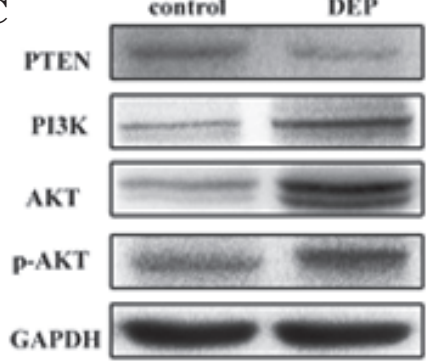

Figure 1. (A) miR-21 expression was 16-fold higher in A549 cells compared with HBE cells. DEPs increased the miR-21 expression by six-fold in the HBE cells. (B) Western blot analysis indicated that PTEN, PI3K, p-AKT and AKT were expressed in the HBE and A549 cells. (C) PTEN expression was suppressed in HBE cells after DEP treatment for $48 \mathrm{~h}$, whereas AKT and PI3K expression were increased simultaneously. HBE, human bronchial epithelial; DEPs, diesel exhaust particles; PTEN, phosphatase and tensin homolog; PI3K, phosphatidylinositol 3'-kinase; p-AKT, phosphorylated AKT; miR, microRNA.

as well as the HBE and A549 cells that were treated with DEPs for $48 \mathrm{~h}$, were removed from the culture flasks and resuspended at $1 \times 10^{5}$ cells $/ \mathrm{ml}$ in serum-free medium. Then, $0.1 \mathrm{ml}$ of the cell suspension was added to the upper chambers. The medium supplemented with serum was used as a chemoattractant in the lower chamber. The chambers were incubated for $48 \mathrm{~h}$ at $37^{\circ} \mathrm{C}$ in a humid atmosphere of $5 \% \mathrm{CO}_{2} / 95 \%$ air. The filters were then fixed in $95 \%$ ethanol and stained with hematoxylin. The upper surfaces of the filters were scraped thrice with cotton swabs to remove the non-invasive cells. The experiments were repeated thrice and the migrated cells were microscopically counted in five different fields per filter.

Wound healing assay. The HBE and A549 cells were cultured in six well plates and the clones were grown to confluency. A linear wound was made by scraping a closed Pasteur pipette across the confluent cell layer, following $24 \mathrm{~h}$ DEP treatment in the experimental group. The cells were washed twice to remove the detached cells and debris. Next, the wounds sizes were observed and measured at set times.

\section{Results}

DEPs increase the miRNA-21 expression in human airway epithelial cells. First, the miR-21 expression in HBE cells, A549 and treated HBE cells was profiled. The analysis demonstrated that miR-21 is overexpressed by greater than 16-fold in untreated A549 cells compared with the untreated HBE cells. The miR-21 expression in the HBE cells treated with DEPs for $48 \mathrm{~h}$ was 6 -fold higher than the untreated HBE cells (Fig. 1A).

DEPs activate the PTEN/PI3K/AKT pathway in HBE cell lines. The effect of DEPs on PTEN/PI3K/AKT activity was then measured. PTEN, PI3K, phosphorylated AKT (p-AKT) and AKT were immunoprecipitated from the total cell extracts derived from the HBE cells treated with $100 \mu \mathrm{g} / \mathrm{ml}$ DEPs. The PTEN, PI3K, p-AKT and AKT expression in untreated and treated HBE cells were examined (Fig. 1B). As determined by western blot analysis, the PTEN expression was significantly reduced in the untreated HBE cells. However, PI3K, AKT and p-AKT expression were markedly increased in the HBE cells following treatment with DEPs for $48 \mathrm{~h}$ (Fig. 1C).

DEPs in combination with miR-21 antisense oligonucleotides (As-miR-21) alter DEP-mediated miR-21 expression. To evaluate the effect of the miR-21 inhibitor on the experimental group treated with DEPs, qPCR was used to compare the expression of miR21 in HBE cells transfected with the miR-21 inhibitor in the experimental and control groups. The measurements were performed $48 \mathrm{~h}$ following transfection. The data were analyzed from triplicate samples. DEPs alone increased the miR-21 expression in the studied cells. However, following transfection of the DEP-treated group with the miR-21 inhibitor, miR-21 expression was significantly reduced (Fig. 2A).

As-miR-21 treatment suppresses activation of the PTEN/PI3K/AKT pathway by DEPs in HBE cell lines. Studies have demonstrated that PTEN is a miR-21 target gene, and PTEN regulates the PI3K/ AKT pathway (16). PTEN frequently inhibits PI3K/AKT pathway activation in lung cancer $(15,17)$. Therefore, one potential function of spliced miR-21 may be to upregulate the PTEN tumor suppressor gene. However, the above analysis demonstrated that increasing the expression of miR-21 suppressed PTEN in HBE cells. Given that the inhibition of gene expression by microRNAs is well understood, as-miR-21 was transfected into the experimental group above, and an increase in the PTEN protein expression and a 
A

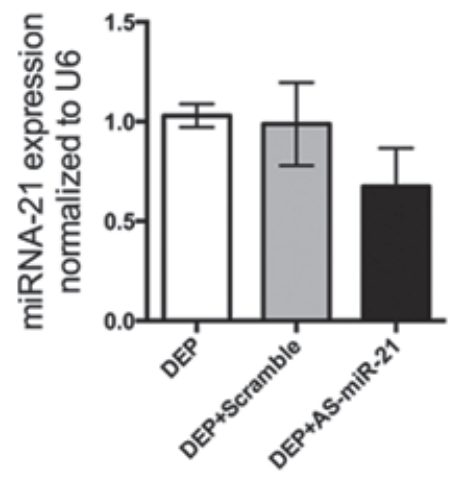

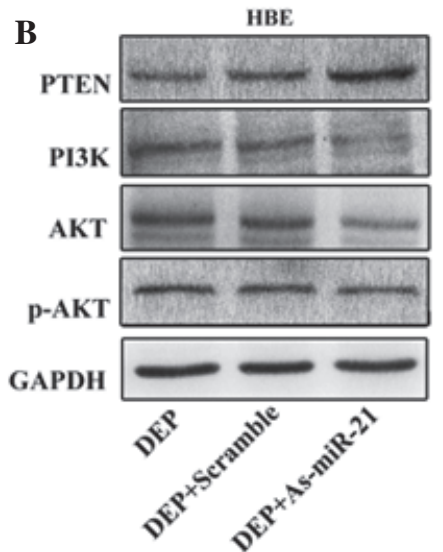

Figure 2. (A) As-miR-21 combined with DEPs induced a marked decrease in miR-21 expression in HBE cells. (B) PTEN, PI3K and AKT expression were monitored by western blot analysis following treatment with DEPs $(100 \mu \mathrm{g} / \mathrm{ml})$ alone or in combination with As-miR-21 in HBE cells for $48 \mathrm{~h}$. HBE, human bronchial epithelial; DEPs, diesel exhaust particles; PTEN, phosphatase and tensin homolog; PI3K, phosphatidylinositol 3'-kinase; p-AKT, phosphorylated AKT; miR, microRNA.

A

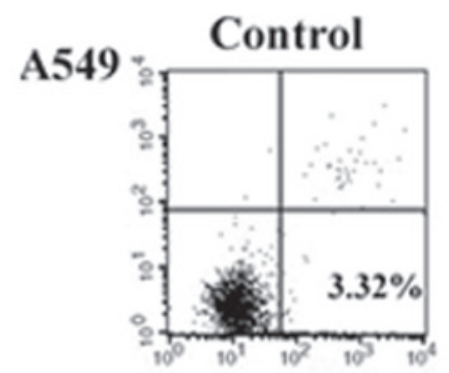

HBE

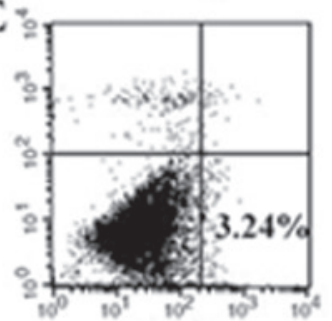

DEP
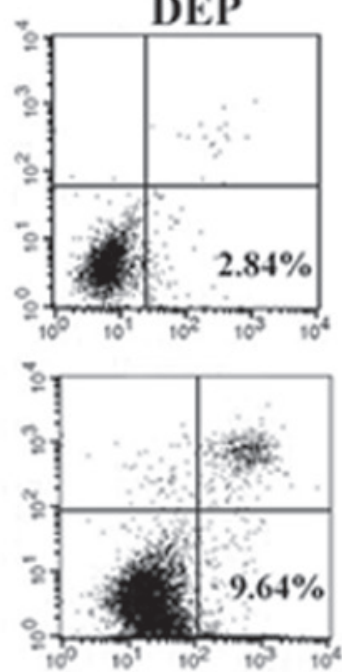

B
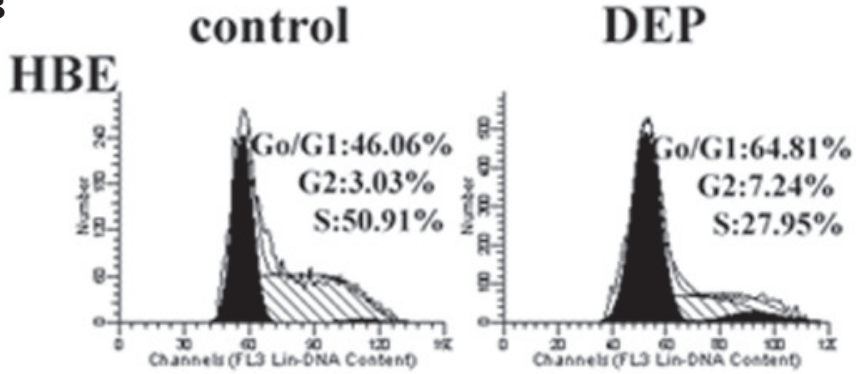

A549

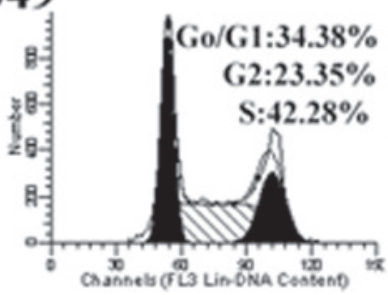

DEP

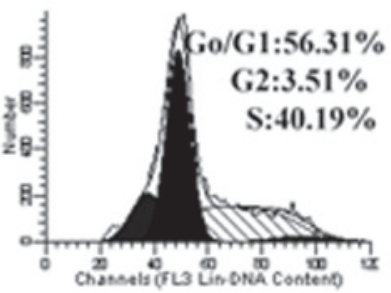

Figure 3. (A) Flow cytometry analyses of propidium iodide-stained A549 cells were performed in triplicate $48 \mathrm{~h}$ following DEP treatment. (B) The A549 and HBE cells were treated with DEP for $48 \mathrm{~h}$ and the cell cycle distributions were detected by flow cytometry. HBE, human bronchial epithelial; DEPs, diesel exhaust particles; miR, microRNA.

reduction in the PI3K, p-AKT and AKT expression in the HBE cells was subsequently observed (Fig. 2B).

DEPs increase apoptosis in HBE cells but not A549 cells. To analyze whether increased miR-21 expression in HBE and A549 cells was able to alter cellular apoptosis, DEP-induced apoptosis was measured via annexin V-PI staining in HBE and A549 cells. The cells were exposed to DEPs $(100 \mu \mathrm{g} / \mathrm{ml})$ for $48 \mathrm{~h}$. As demonstrated in Fig. 3A and B, a significant increase in early phase apoptotic cells was observed in the DEP-treated HBE cells (9.64\%) compared with the untreated cells (3.24\%) $(\mathrm{P}<0.05)$; however, no significant differences were observed between the treated (2.84\%) and untreated A549 cells (3.32\%; $\mathrm{P}>0.05$; Fig. 3A).

DEPs induce G0/G1 arrest in HBE and A549 cells. DEP has been reported to induce growth arrest at the G1/G0 phase in a wide variety of cells (18-20). To understand the effects of DEP treatment on cell growth in the present study, the cell cycle distribution of HBE and A549 cells $48 \mathrm{~h}$ following treatment with $100 \mu \mathrm{g} / \mathrm{ml}$ DEPs was measured via PI staining. The untreated cells served as negative controls. DEPs induce a significant increase $(\mathrm{P}<0.05)$ in the number of $\mathrm{S}$-phase cells in the A549 cell lines. DEPs markedly enhanced the number of G0/G1-phase cells in HBE cells ( $\mathrm{P}<0.05$; Fig. 3B).

DEPs alter HBE cell migration and invasion, but have a weak effect on A549 cells. To examine the functional consequences of DEPs in HBE cells and A549 cell lines, the Transwell assay was used to determine cell invasiveness and wound healing assays were used to detect cell motility in the cells treated with DEPs $(100 \mu \mathrm{g} / \mathrm{ml})$. Significant differences in the number of invasive cells were observed between the control and experimental groups (Fig. 4A). In addition, evident differences in the 
A
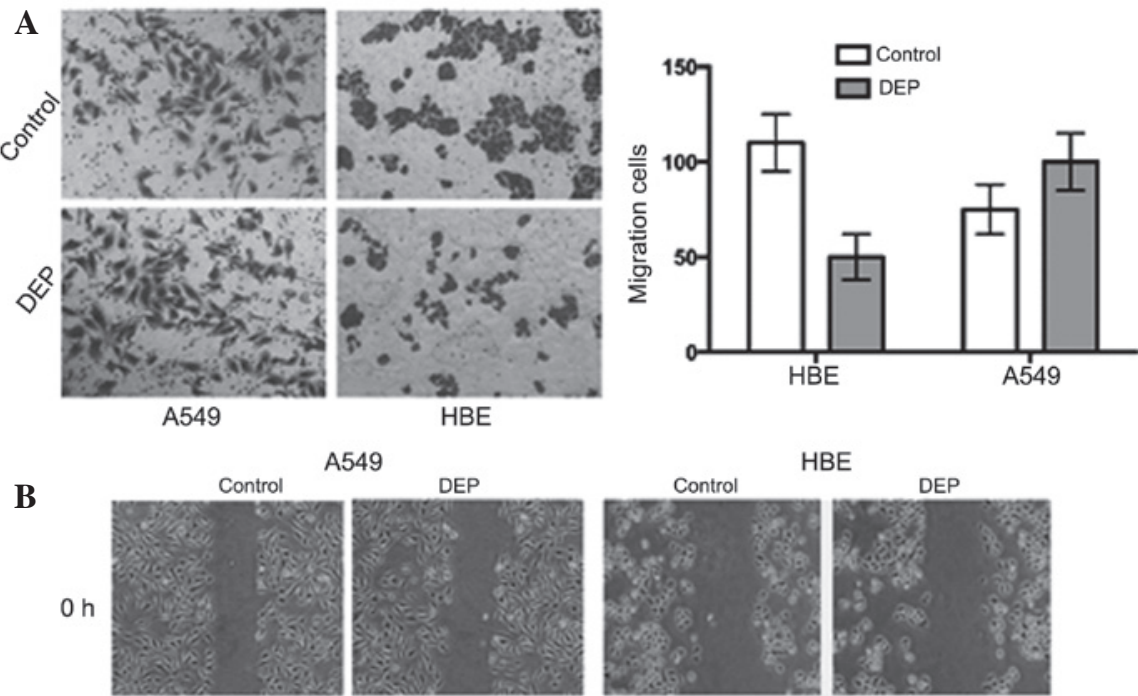

A549
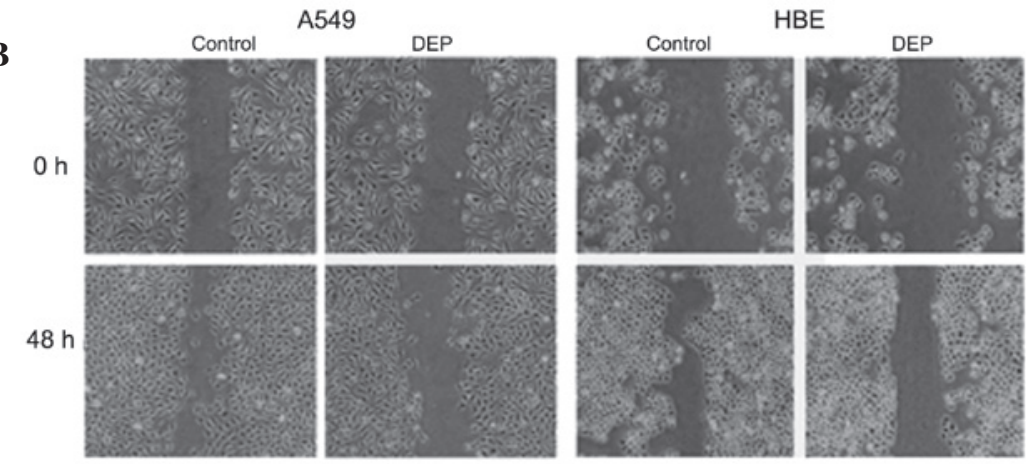

C
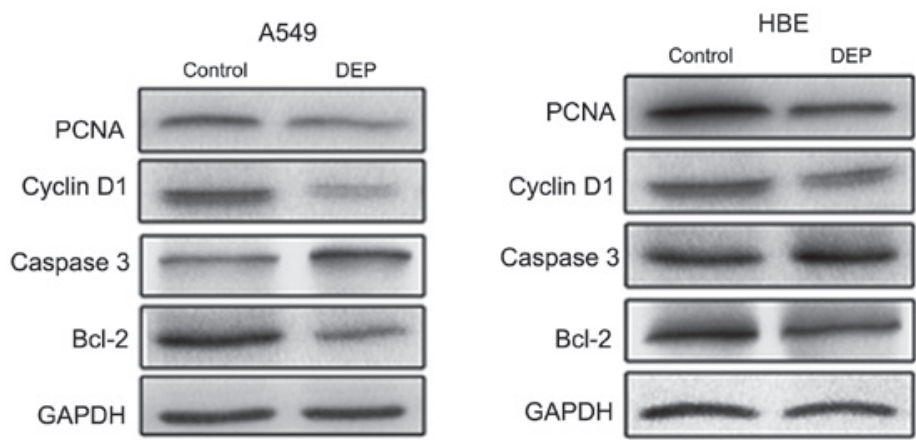

Figure 4. (A) Cell invasion was assessed by a transwell assay following DEP treatment for 48 h. (B) The HBE and A549 cell monolayers were wounded by scraping with a closed Pasteur pipette and the wound size was measured $48 \mathrm{~h}$ later. (C) The effect of DEPs on Bcl-2, caspase-3, PCNA and cyclin D1 expression in HBE and A549 cells was analyzed by western blotting following 48 h DEP treatment. HBE, human bronchial epithelial; DEPs, diesel exhaust particles; miR, microRNA.

cellular migration remained between the treated cells and the control samples. A significant portion of HBE cells invaded the lower chamber $(\mathrm{P}<0.05)$, however this phenomenon was not observed in the A549 cells ( $\mathrm{P}>0.05)$. Cell motility was suppressed by DEPs in the experimental group in HBE and A549 cells $(\mathrm{P}<0.05)$, but the effect was less prominent in A549 cells compared with HBE cells (Fig. 4B).

\section{Discussion}

In light of economic development and industrialization, there is an increasing concern regarding air pollutants, particularly PM2.5 (21) (particulate matter with an aerodynamic diameter $\leq 2.5 \mu \mathrm{m}$ ). Various studies have indicated that increases in lung cancer mortality are associated with PM2.5 exposure $(22,23)$. DEPs, an important component of air pollution, is another research topic that has attracted notable attention. Lung cancer remains one of the most frequently occurring malignancies and numerous studies have demonstrated that DEPs may promote lung carcinogenesis $(4,7,8,24)$. Therefore, the study of the molecular mechanisms underlying the effects

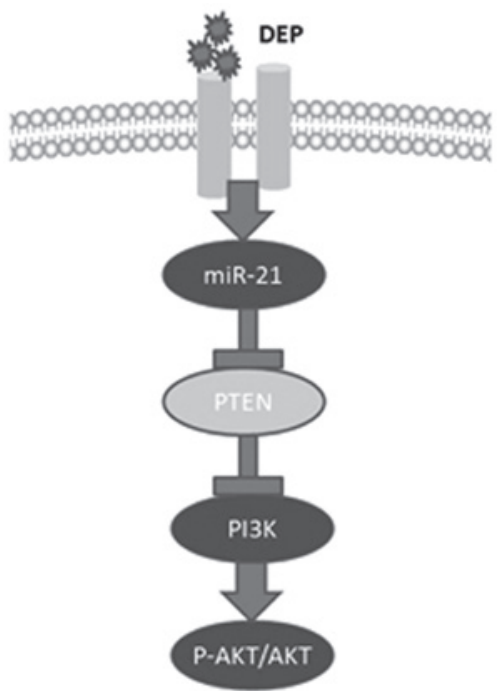

Figure 5. The potential carcinogenic mechanism: DEPs increase miR-21 expression and then activate the classical PTEN/PI3K/AKT pathway. HBE, human bronchial epithelial; DEPs, diesel exhaust particles; PTEN, phosphates and tensin homolog; PI3K, phosphatidylinositol 3'-kinase; p-AKT, phosphorylated AKT; miR, microRNA. 
of DEPs in lung carcinogenesis remains a topic worthy of investigation.

miR-21 is a unique miRNA highly expressed in a variety of solid tumor types, including lung, stomach, prostate, colon, esophageal, pancreatic and cervical cancer. With regard to lung adenocarcinoma (25-27), miR-21 expression is increased 16 -fold compared with normal tissue. Several studies have demonstrated that miR-21 expression may serve as diagnostic criteria in lung cancer (28). The carcinogenic mechanism of DEPs may be associated with increased expression of the suppressor gene, PTEN, in tumors $(29,30)$. In the present study, miR-21 oncogenic expression was 16-fold higher in the A549 cells than in the HBE cells. Following DEP treatment, miR-21 expression increased 6-fold in the HBE cells; this expression level was similar in the A549 cells. Therefore, it was hypothesized that DEPs increase the miR-21 expression in a process that may be associated with the potential mechanisms of carcinogenesis.

PTEN, also known as MMAC1 (mutated in multiple advanced cancers), is a tumor suppressor gene with sequence homology to tyrosine phosphatases and the cytoskeletal proteins tensin and auxilin (31). PTEN is an important miR-21 target gene. Among its numerous activities, PTEN is involved in the integration of proteins during cell growth regulation, tumor invasion, angiogenesis and metastasis. Numerous studies have demonstrated that PTEN is an important signaling molecule in the PTEN/PI3K/AKT signal transduction pathways (16). The loss or decrease of PTEN activity promotes the survival of NSCLC cells (15). However, certain studies suggest that PTEN tumor suppressor function is exerted through negative regulation of the PI3K/AKT cell survival pathway (17). Jardim et al (15) treated HBE cells with suspended DEPs and the total RNA was isolated for the microarray analysis $24 \mathrm{~h}$ following treatment. This study revealed that 197 of 313 detectable miRNAs $(62.9 \%)$ were either upregulated or downregulated by 1.5 -fold. Furthermore, the expression of PTEN products was significantly inhibited with the exception of PI3K and AKT, which exhibited increased expression (15). In the present study, the expression of PTEN, PI3K, p-AKT and AKT between untreated HBE and A549 cells was first compared. The PTEN expression in A549 cells was evidently reduced compared with HBE cells. However, PI3K and p-AKT exhibited an opposing effect. PTEN expression decreased in the HBE cells following DEP treatment for $48 \mathrm{~h}$, whereas PI3K and AKT expression markedly increased. The A549 cells exhibited expression patterns similar to those observed in the treated HBE cells. When the cells were treated with DEP in combination with miR-21 inhibitors, opposite results were obtained. PTEN expression increased, whereas PI3K and AKT expression decreased. When miR-21 expression was suppressed by the miR-21 inhibitor, the expression of PTEN, PI3K, AKT subsequently changed. Considering that PTEN is an important miR-21 target, it was concluded that DEPs increase miR-21 expression and subsequently activates the PI3K/AKT pathway by reducing PTEN expression, which may be the mechanism that underlies the potential carcinogenic effects of DEPs.

Limitless replicative potential and the evasion of apoptosis are two critical factors in tumorigenesis (32). Numerous studies have demonstrated that strong, short-term DEP treatment in HBE cells results in increased apoptosis and decreased proliferation index drop, as well as cell cycle inhibition at G0/G1 phase (18-20). In the present study, a similar result was obtained following treatment of the HBE cells with DEPs (Fig. 4C). Increased caspase 3 expression and modest Bcl-2, cyclin D1 and proliferation index PCNA expression in the experimental group was also observed, consistent with the results of previous studies. In addition, the present study also assessed the above-mentioned parameters in A549 lung adenocarcinoma cells and it was identified that the experimental group exhibited results similar to the HBE cells. However, the apoptosis rate was not significantly altered in the A549 cells. These findings suggest that exposure to high concentrations of DEPs in a short time did not cause evident cancer progression in HBE cell morphology. DEP exposure also did not cause deteriorating A549 cell morphology.

The present results evidently demonstrate the impact of DEP exposure on miR-21 expression in HBE cells. DEP-induced alterations in miR-21 expression may potentially have an important role in the development and progression of primary lung carcinoma. Further studies on DEP-induced alterations in PTEN, PI3K and AKT expression, as well as DEP in combination with a miR-21 antisense oligonucleotide, may allow for a more comprehensive study of DEP-induced pathogenic states and underlying molecular mechanisms of potential carcinogenicity. However, the present study only examined a single stimulus and its short-term effect on cell morphology and the PTEN/PI3K/AKT pathway. In addition, the stimulus concentration was notably greater than the concentration that people are exposed to in real life. The increased levels of stimulation may cause a series of destructive changes in cell morphology in a short period of time, including alterations in proliferation, invasion and migration. Wu et al found that DEPs impair cell viability in a dose- and time-dependent manner (33). However, the concentration of DEPs in daily life is notably lower than the concentration used in the experiment. Based on repair capability in the cell, the concentration of DEPs in real life would not be as damaging to cells. Using DEPs as a single stimulus, the classic carcinogenic PTEN/PI3K/AKT pathway was activated. Therefore, it was hypothesized that long-term exposure to low DEP concentrations may gradually activate the PTEN/PI3K/AKT pathway, thereby acting as a potential carcinogen (Fig. 5). Briefly, it is concluded that DEPs are involved in human lung carcinogenesis and that one of the potential carcinogenic mechanisms of DEP involves increased miR-21 expression. Increased miR-21 expression results in decreased PTEN activity, consequently activating the classic carcinogenic PI3K/AKT pathway and then promoting carcinogenesis.

The environmental modulation of gene expression is the focus of intense study, but how DEPs cause lung cancer remains unanswered. Overall, further studies are required to focus on additional miRNAs and signal transduction pathways altered by diesel exhaust particles or other pollutants involved in lung carcinogenesis. Therefore, studies investigating the effects of the environment on cancer remain an important priority.

\section{Acknowledgements}

This study was supported by the State Key Laboratory of Internal Combustion Engine Fuel Science of Tianjin University, 
open project 2011 (K2011-04). The authors are grateful to the members of the Tianjin Laboratory of Neuro-Oncology, Tianjin Neurological Institute for their technical assistance (Tianjin, China).

\section{References}

1. Jemal A, Center MM, DeSantis C, et al: Global patterns of cancer incidence and mortality rates and trends. Cancer Epidemiol Biomarkers Prev 19: 1893-1907, 2010.

2. Ramalingam SS, Owonikoko TK, et al: Lung cancer: New biological insights and recent therapeutic advances. CA Cancer J Clin 61: 91-112, 2011.

3. Grant WB: Air pollution in relation to U.S. cancer mortality rates: an ecological study; likely role of carbonaceous aerosols and polycyclic aromatic hydrocarbons. Anticancer Res 29: 3537-3545, 2009.

4. Silverman DT, Samanic CM, Lubin JH, et al: The Diesel Exhaust in Miners study: a nested case-control study of lung cancer and diesel exhaust. J Natl Cancer Inst 104: 855-868, 2012.

5. Villeneuve PJ, Parent MÉ, Sahni V, et al: Occupational exposure to diesel and gasoline emissions and lung cancer in Canadian men. Environ Res 111: 727-735, 2011.

6. Chang CC, Tsai SS, Chiu HF, et al: Traffic air pollution and lung cancer in females in Taiwan: petrol station density as an indicator of disease development. J Toxicol Environ Health A 72: 651-657, 2009.

7. Laumbach RJ and Kipen HM: Does diesel exhaust cause lung cancer (yet)? Am J Respir Crit Care Med 183: 843-844, 2011.

8. Attfield MD, Schleiff PL, Lubin JH, et al: The Diesel Exhaust in Miners study: a cohort mortality study with emphasis on lung cancer. J Natl Cancer Inst 104: 869-883, 2012.

9. Hou L, Wang D and Baccarelli A: Environmental chemicals and microRNAs. Mutat Res 714: 105-112, 2011.

10. Yang L, Li N, Wang $\mathrm{H}$, et al: Altered microRNA expression in cisplatin-resistant ovarian cancer cells and upregulation of miR-130a associated with MDR1/P-glycoprotein-mediated drug resistance. Oncol Rep 28: 592-600, 2012.

11. Tao J, Lu Q, Wu D, et al: microRNA-21 modulates cell proliferation and sensitivity to doxorubicin in bladder cancer cells Oncol Rep 25: 1721-1729, 2011.

12. Gao W, Lu X, Liu L, et al: MiRNA-21: a biomarker predictive for platinum-based adjuvant chemotherapy response in patients with non-small cell lung cancer. Cancer Biol Ther 13: 330-340, 2012.

13. Iorio MV, Ferracin M, Liu CG, et al: MicroRNA gene expression deregulation in human breast cancer. Cancer Res 65: 7065-7070, 2005.

14. Ma C, Wang J and Luo J: Activation of nuclear factor kappa B by diesel exhaust particles in mouse epidermal cells through phosphatidylinositol 3-kinase/Akt signaling pathway. Biochem Pharmacol 67: 1975-1983, 2004.

15. Jardim MJ, Fry RC, Jaspers I, et al: Disruption of microRNA expression in human airway cells by diesel exhaust particles is linked to tumorigenesis-associated pathways. Environ Health Perspect 117: 1745-1751, 2009.

16. Kandasamy K and Srivastava RK: Role of the phosphatidylinositol 3'-kinase/PTEN/Akt kinase pathway in tumor necrosis factor-related apoptosis-inducing ligand-induced apoptosis in non-small cell lung cancer cells. Cancer Res 62: 4929-4937, 2002.
17. Shu H, Zhang $\mathrm{H}, \mathrm{Xu} \mathrm{C}$, et al: Clinicopathological research and expression of PTEN/PI3K/Akt signaling pathway in non-small cell lung cancer. Zhongguo Fei Ai Za Zhi 12: 889-892, 2009 (In Chinese).

18. Doornaert B, Leblond V, Galiacy S, et al: Negative impact of DEP exposure on human airway epithelial cell adhesion, stiffness, and repair. Am J Physiol Lung Cell Mol Physiol 284: L119-L132, 2003.

19. Li N, Wang M, Oberley TD, et al: Comparison of the pro-oxidative and proinflammatory effects of organic diesel exhaust particle chemicals in bronchial epithelial cells and macrophages. J Immunol 169: 4531-4541, 2002.

20. Hiura TS, Kaszubowski MP, Li N, et al: Chemicals in diesel exhaust particles generate reactive oxygen radicals and induce apoptosis in macrophages. J Immunol 163: 5582-5591, 1999.

21. Chuang KJ, Chuang HC and Lin LY: Indoor air pollution, nighttime heart rate variability and coffee consumption among convenient store workers. PLoS One 8: e63320, 2013.

22. Vinikoor-Imler LC, Davis JA and Luben TJ: An ecologic analysis of county-level PM2.5 concentrations and lung cancer incidence and mortality. Int J Environ Res Public Health 8: 1865-1871, 2011.

23. Hystad P, Demers PA, Johnson KC, et al: Long-term residential exposure to air pollution and lung cancer risk. Epidemiology 24: 762-772, 2013.

24. Olsson AC, Gustavsson P, Kromhout H, et al: Exposure to diesel motor exhaust and lung cancer risk in a pooled analysis from case-control studies in Europe and Canada. Am J Respir Crit Care Med 183: 941-948, 2011.

25. Furuta C, Suzuki AK, Watanabe G, et al: Nitrophenols isolated from diesel exhaust particles promote the growth of MCF-7 breast adenocarcinoma cells. Toxicol Appl Pharmacol 230: 320-326, 2008.

26. Møller P, Folkmann JK, Danielsen PH, et al: Oxidative stress generated damage to DNA by gastrointestinal exposure to insoluble particles. Curr Mol Med 12: 732-745, 2012.

27. Raaschou-Nielsen O, Andersen ZJ, Hvidberg M, et al: Air pollution from traffic and cancer incidence: a Danish cohort study. Environ Health 10: 67, 2011.

28. Rabinowits G, Gerçel-Taylor C, Day JM, et al: Exosomal microRNA: a diagnostic marker for lung cancer. Clin Lung Cancer 10: 42-46, 2009.

29. Zhang JG, Wang JJ, Zhao F, et al: MicroRNA-21 (miR-21) represses tumor suppressor PTEN and promotes growth and invasion in non-small cell lung cancer (NSCLC). Clin Chim Acta 411: 846-852, 2010.

30. Zhang W, Bai W and Zhang W: MiR-21 suppresses the anticancer activities of curcumin by targeting PTEN gene in human non-small cell lung cancer A549 cells. Clin Transl Oncol: Nov 29, 2013 (Epub ahead of print).

31. Hong TM, Yang PC, Peck K, et al: Profiling the downstream genes of tumor suppressor PTEN in lung cancer cells by complementary DNA microarray. Am J Respir Cell Mol Biol 23: 355-363, 2000.

32. Hanahan D and Weinberg RA: The hallmarks of cancer. Cell 100: 57-70, 2000.

33. Wu Y, Yu T, Gilbertson TA, et al: Biophysical assessment of single cell cytotoxicity: diesel exhaust particle-treated human aortic endothelial cells. PLoS One 7: e36885, 2012. 